TRANSACTIONS OF THE

AMERICAN MATHEMATICAL SOCIETY

Volume 279, Number 2, October 1983

\title{
LINEARIZATION OF SECOND-ORDER NONLINEAR OSCILLATION THEOREMS
}

BY

MAN KAM KWONG ${ }^{1}$ AND JAMES S. W. WONG

\begin{abstract}
The problem of oscillation of super- and sublinear Emden-Fowler equations is studied. Established are a number of oscillation theorems involving comparison with related linear equations. Recent results on linear oscillation can thus be used to obtain interesting oscillation criteria for nonlinear equations.
\end{abstract}

1. We are concerned here with the oscillatory behaviour of continuable solutions of the following second-order nonlinear differential equation on $[0, \infty)$;

$$
y^{\prime \prime}(t)+a(t) f(y(t))=0, \quad t \in[0, \infty),
$$

where $a(t)$ is piecewise continuous and $f$ is a continuously differentiable function on $(-\infty, \infty)$ such that

$$
y f(y)>0 \text { for } y \neq 0, \text { and } f^{\prime}(y) \geqslant 0 .
$$

The prototype of (1) is the so-called generalized Emden-Fowler equation

$$
y^{\prime \prime}(t)+a(t)|y(t)|^{\gamma} \operatorname{sgn} y(t)=0,
$$

where $\gamma>0$ is a positive constant. Equation (3) is called superlinear if $\gamma>1$ and sublinear if $0<\gamma<1$. We call an equation oscillatory if all its continuable solutions are oscillatory.

When $\gamma \neq 1$ and $a(t)$ is nonnegative, Atkinson [1] and Belohorec [3] established necessary and sufficient conditions for the oscillation of (3). When $a(t)$ is allowed to take negative values, the conditions of Atkinson and Belohorec are known to be sufficient for oscillation, see Kiguradze [18] and Belohorec [4]. Recently, these results have been further extended by Kwong and Wong [21]. Unfortunately, these results are effective only in cases when a certain integral involving the coefficient $a(t)$ diverges and are not very effective in dealing with the prototype cases when $a(t)=t^{\lambda} \sin t, t^{\lambda} \cos t$ or more generally $t^{\lambda} \phi(t)$ where $\phi(t)$ is periodic of mean value zero. See recent results by Butler $[\mathbf{5}, \mathbf{6}, \mathbf{8}]$ and Kwong and Wong [21, 22].

A different approach is to relate the oscillation problem of (3), or more generally (1), to that of some linear second-order differential equation. There is a vast literature on linear oscillation; see for example Hartman [15], Barrett [2], Rab [31],

Received by the editors February 5, 1982 and, in revised form, November 21, 1982.

1980 Mathematics Subject Classification. Primary 34C10, 34C15.

Key words and phrases. Second order, nonlinear, differential equations, oscillation.

'This work was supported in part by the Applied Mathematical Sciences Research Program (KC-04-02) of the Office of Energy Research of the U. S. Department of Energy under Contract W-31-109-ENG-38. 
Coppel [11], Wong [38,39], Willett [36] and the many references therein. In spite of such a voluminous collection of oscillation results, some new and fairly general results have recently been developed in Kwong [20] and Kwong and Zettl [24, 25]. The purpose of this paper is to relate these results to that of the second-order nonlinear equation (3) or more generally (1) by way of comparison.

2. The first results relating nonlinear oscillation of (3) to that of the linear equation

$$
x^{\prime \prime}(t)+a(t) x(t)=0, \quad t \in[0, \infty),
$$

could be found in Utz [33]. Equation (4) is called strongly oscillatory if the equation

$$
x^{\prime \prime}(t)+c a(t) x(t)=0
$$

is oscillatory for every positive constant $c$. Let us, for later use, call (4) weakly oscillatory if the above equation is oscillatory for some positive constant $c$. Notice that for the Emden-Fowler equation (3), $\gamma \neq 1$, the concepts of strong oscillation, oscillation and weak oscillation are equivalent, because solutions of

$$
y^{\prime \prime}(t)+c a(t)|y(t)|^{\gamma} \operatorname{sgn} y(t)=0
$$

are simply multiples of those of (3).

Unfortunately, Utz's results on oscillation were rather fragmentary. More complete results may be found in Erbe [13] and Wong [41].

Theorem A (Wong [41]). Suppose that $f(y)$ satisfies (2) and there is a positive constant $c$ such that $f^{\prime}(y) \geqslant c>0$ for all $y \in(-\infty, \infty)$. If, for this fixed $c$, (4) is oscillatory, then (1) is also oscillatory.

THEOREM B (ERBE [12]). Suppose that the function $f(y)$ satisfies

$$
f^{\prime}(y) \geqslant f(y) / y>0 \text { for } y \neq 0,
$$

(note that condition (5) implies (2)) and that a( $t$ ) satisfies

$$
\liminf _{t \rightarrow \infty} \int_{T}^{t} a(s) d s \geqslant 0
$$

for all large $T$. Then if the linear equation (4) is strongly oscillatory, so is (1).

We shall derive other theorems of the same sort in the next three sections. Let us first see how Theorem A follows from the classical Picone-Sturm Comparison Theorem for the linear equation. The same arguments prevail in many of the proofs in the rest of the paper. Let $y(t)$ be a nonoscillatory solution, then $r(t)=$ $-y^{\prime}(t) / f(y(t))$ satisfies the Riccati differential equation

$$
r^{\prime}(t)=a(t)+f^{\prime}(y(t)) r^{2}(t), \quad t \in\left[t_{0}, \infty\right),
$$

for some $t_{0} \geqslant 0$. If we let $p(t)=1 / f^{\prime}(y(t))$, then the Riccati equation

$$
r^{\prime}(t)=a(t)+r^{2}(t) / p(t)
$$

has a solution on $\left[t_{0}, \infty\right)$. It is well known that this is equivalent to the nonoscillation of the linear equation

$$
\left(p(t) z^{\prime}(t)\right)^{\prime}+a(t) z(t)=0, \quad t \geqslant 0 .
$$


By assumption, $p(t) \geqslant 1 / c$, and applying the Picone-Sturm Theorem to the following equation

$$
\left(x^{\prime}(t) / c\right)^{\prime}+a(t) x(t)=0
$$

we conclude that it is nonoscillatory, contradicting the hypothesis.

Theorem B can be proved along similar lines. First, it follows from (5) that for $0<y_{1}<y_{2}$,

$$
\int_{y_{1}}^{y_{2}} \frac{f^{\prime}(y)}{f(y)} d y \geqslant \int_{y_{1}}^{y_{2}} \frac{d y}{y},
$$

or $f^{\prime}\left(y_{2}\right) \geqslant f\left(y_{1}\right) / y_{1}$. The importance of condition (6) is its implication that any eventually positive (negative) solution of (1) must be eventually nondecreasing (nonincreasing) as proved by Erbe [12]. This also follows from results in our earlier work [41, Lemma 2], see also Kwong and Wong [23], which follows a completely different approach. Hence if (1) has a solution $y(t)$ which is positive on $\left[t_{0}, \infty\right)$, then $f^{\prime}(y(t)) \geqslant f\left(y\left(t_{0}\right)\right) / y\left(t_{0}\right)=c>0$, for all $t \in\left[t_{0}, \infty\right)$. The arguments used in proving Theorem A can now be repeated to complete the proof.

Let us point out that an application of Theorem A gives the following new oscillation criteria for (1).

Corollary 1. Let $f(y)$ satisfy (2) and let there be a positive constant $c>0$ such that $f^{\prime}(y) \geqslant c$ for all $y \in(-\infty, \infty)$. Suppose that a $(t)$ satisfies

$$
\limsup _{T \rightarrow \infty} \frac{1}{T} \int_{0}^{T} \int_{0}^{t} a(s) d s d t=+\infty,
$$

and that there exists a constant $b>0$ such that

$$
\int_{t_{0}}^{t} a(s) d s \geqslant-e^{h t}, \quad t \geqslant t_{0},
$$

for some $t_{0} \geqslant 0$, then (1) is oscillatory.

This result generalizes a theorem of Onose [28, Theorem 3, p. 71], where condition (10) is replaced by the stronger assumption

$$
\liminf _{t \rightarrow \infty} \int_{0}^{t} a(s) d s \geqslant-L>-\infty
$$

and $f(y)$ satisfies an additional nonlinearity condition (which incidentally rules out the linear case as well). To see how Corollary 1 follows from Theorem A, we need only invoke a theorem of Putnam [30] which shows that (9) and (10) are sufficient for (4) to be strongly oscillatory. Similarly, results of Kamenev [17] extending those weighted average theorems of Willett [35] for linear oscillation follow readily from Theorem A; also see Butler [8].

3. We note that the condition imposed on $f(y)$ in Theorem $\mathrm{A}$ is very restrictive as it excludes the important case of (3). Likewise, condition (6) on $a(t)$ in Theorem B is rather limited in its applications. Indeed, if (6) holds then $\lim _{T \rightarrow \infty} \int_{0}^{T} a(t) d t$ exists and in case it is finite, $\int_{t}^{\infty} a(s) d s \geqslant 0$ for all large $t$ (see Coles [9,p. 127]). Thus, $a(t)=t^{\lambda} \sin t, \lambda \in(-\infty, \infty)$, does not satisfy (6). Indeed the classification of the 
Emden-Fowler equation with respect to oscillation for $a(t)=t^{\lambda} \sin t$ is by no means easy, see Butler [5,6,8], Wong [41], Kwong and Wong [21, 22]. The purpose of this paper is to present new linearization results of the type discussed above which are effective in resolving these prototype cases, thereby offering alternative means of classifying such equations with respect to oscillation. For the sake of simpler exposition, we shall state and prove our results for (3). It would become obvious from the proofs how some of these results in the sequel can be extended to the more general equation (1) under suitable assumptions on the nonlinear function $f(y)$. We refer the reader to [41] for a collection of such conditions.

Our first result concerns superlinear equations with integrable coefficients, i.e., when

$$
\lim _{T \rightarrow \infty} \int_{0}^{T} a(s) d s \text { exists and is finite. }
$$

We define $A(t)=\int_{t}^{\infty} a(s) d s$ and

$$
p(t)=\max \left\{1,-\int_{0}^{t} A(s) d s\right\} .
$$

THEOREM 1. Suppose that the linear equation

$$
\left(p(t) z^{\prime}\right)^{\prime}+a(t) z=0
$$

is strongly oscillatory, i.e. $\left(p(t) z^{\prime}\right)^{\prime}+c a(t) z=0$ is oscillatory for every $c>0$; then (3) is oscillatory with $\gamma>1$.

Proof. We make use of an analog of a theorem of Wintner established in our earlier paper [23, Theorem 1]. If (3) has a nonoscillatory solution $y(t)$ which may be assumed to be positive on $\left[t_{0}, \infty\right)$, then $y(t)$ satisfies the integral equation

$$
\frac{y^{\prime}(t)}{y^{\gamma}(t)}=A(t)+\gamma \int_{t}^{\infty} \frac{y^{\prime 2}(s)}{y^{\gamma+1}(s)} d s .
$$

By integrating the above, we find that

$$
\frac{1}{y^{\gamma-1}\left(t_{0}\right)}-\frac{1}{y^{\gamma-1}(t)} \geqslant(\gamma-1) \int_{t_{0}}^{t} A(s) d s,
$$

from which it follows

$$
y^{\gamma-1}(t) \geqslant\left[(\gamma-1)\left(c_{0}-\int_{0}^{t} A(s) d s\right)\right]^{-1} \geqslant \frac{\delta}{p(t)}
$$

where

$$
c_{0}=\frac{y^{1-\gamma}\left(t_{0}\right)}{\gamma-1}+\int_{0}^{t_{0}} A(s) d s
$$

and $\delta>0$ is any positive number less than $\left[(\gamma-1)\left(c_{0}+1\right)\right]^{-1}$. Now let $r(t)=$ $-y^{\prime}(t) / y^{\gamma}(t)$. Then $r(t)$ satisfies the Riccati equation

$$
r^{\prime}(t)=a(t)+\gamma y^{\gamma-1}(t) r^{2}(t),
$$

and hence the corresponding linear equation

$$
\left(y^{1-\gamma}(t) u^{\prime}(t)\right)^{\prime}+\gamma a(t) u(t)=0
$$


is nonoscillatory. By (15) and an application of the Picone-Sturm Comparison Theorem to (16), we conclude that $\left(p(t) z^{\prime}\right)^{\prime}+\gamma \delta a(t) z=0$ is nonoscillatory, contradicting the given hypothesis.

Corollary 2. Suppose that (12) holds, $A(t)=\int_{t}^{\infty} a(s) d s$ satisfies

$$
\liminf _{t \rightarrow \infty} \int_{0}^{t} A(s) d s>-\infty
$$

and the linear equation (4) is strongly oscillatory, then (3) is oscillatory with $\gamma>1$.

Proof. Condition (17) implies that the function $p(t)$ as defined by (13) is bounded above. Thus, the estimate (15) can be replaced by $y^{\gamma-1}(t) \geqslant \delta_{1}>0$. The same argument will then lead to the desired contradiction.

COROllary 3. Suppose that (17) holds as in Corollary 2 and assume that there exists a positive nondecreasing function $\phi \in C^{1}[0, \infty)$ satisfying

$$
\int^{\infty} \frac{\phi^{\prime 2}}{\phi}<\infty \text { and } \lim _{T \rightarrow \infty} \int_{0}^{T} \phi(s) a(s) d s=\infty .
$$

Then (3) is oscillatory when $\gamma>1$.

That condition (18) implies the strong oscillation of (4) is first proved in Wong [41]. Corollary 3 is a nonlinear extension of Zlamal's Theorem for the linear case $\gamma=1$ and $\phi(t)=t^{\alpha}, 0 \leqslant \alpha<1$. Compare this with Kiguradze's extension [18] of Atkinson's result [1] for the superlinear equation when $\gamma>1$; and the recent result of the authors [21] extending the sublinear counterpart by Belohorec. For further discussions of Zlamal's Theorem in the linear case we refer to Macki and Wong [26], Kwong and Zettl [24].

Since for $\gamma=1$, condition (18) alone without (17) is sufficient for oscillation, we speculate that the same is also true for $\gamma>1$.

EXAmple 1. Consider $a(t)=t^{\lambda} \sin t, \lambda<0$, which clearly satisfies (17). By the result on linear oscillation with integrable coefficients see $[35,39,24$ or 23 , we know that $v^{\prime \prime}+\left(t^{\lambda} \sin t\right) v=0$ is strongly oscillatory if $\lambda>-1$; so does equation (3) in the case when $\gamma>1$.

This result was also covered by a strong oscillation theorem due to Butler [5] who proved that if $A(t)$ satisfies (17)

$$
\lim _{T \rightarrow \infty} \int_{0}^{T}\left(A(t)+\int_{t}^{\infty} A_{+}^{2}(s) d s\right) d t=\infty
$$

implies that (3) is oscillatory with $\gamma>1$. Here $A_{+}(s)=\max \{A(s), 0\}$. Condition (19) shows that (3) with $\gamma>1$ and $a(t)=t^{\lambda} \sin t$ is oscillatory if $\lambda \geqslant-1$. In fact, Butler [8] showed that $\lambda \geqslant-1$ is a necessary and sufficient condition for oscillation of (3) in this case. Proofs of Butler's results are rather lengthy and sophisticated. See Kwong and Wong [23] for an alternative proof and further discussions of the first result of Butler mentioned above. It can be shown by examples that Corollary 2 and Butler's result are independent.

The following alternative formulation of Theorem 1 may be more convenient for practical applications. 
THEOREM 1'. If there exist a positive function $P(t)$ on $[0, \infty)$ and positive constants $c_{1}, c_{2}$ such that for all $t$ large enough, $\int_{0}^{t} A(s) d s \geqslant-c_{1} P(t), P(t) \geqslant c_{2}>0$, then the strong oscillation of $\left(P Z^{\prime}\right)^{\prime}+a Z=0$ implies the oscillation of (3) with $\gamma>1$.

Our next result applies to both sublinear and superlinear equations and requires only that $\int_{0}^{t} a(s) d s$ is bounded below instead of the existence of $\lim _{T \rightarrow \infty} \int_{0}^{T} a(t) d t$. However, in the sublinear case $(0<\gamma<1)$, condition (20) below can be greatly relaxed. See Theorem 6.

THEOREM 2. Suppose that

$$
\liminf _{t \rightarrow \infty} \int_{0}^{t} a(s) d s>-\infty .
$$

If the linear equation

$$
\left(t z^{\prime}(t)\right)^{\prime}+c a(t) z(t)=0,
$$

is weakly oscillatory, i.e. there exists a positive constant c such that (21) is oscillatory, then (3) is oscillatory for $\gamma>0$.

Proof. Suppose that (3) has a solution $y(t)$ which is positive on $\left[t_{0}, \infty\right)$. By our earlier result [23, Corollary 4], condition (20) implies that

$$
\int_{t_{0}}^{\infty} \frac{y^{\prime 2}(s)}{y^{\gamma+1}(s)} d s<\infty .
$$

We claim that

$$
\lim _{t \rightarrow \infty} \frac{1}{t y^{\gamma-1}(t)}=0
$$

Let $t>T \geqslant t_{0}$. Define $\beta=(1-\gamma) / 2$; thus $1-\beta=(\gamma+1) / 2$. Note that by the Schwarz inequality

$$
\begin{aligned}
y^{\beta}(t)-y^{\beta}(T) & \leqslant|\beta| \int_{T}^{t}\left|\frac{y^{\prime}(s)}{y^{1-\beta}(s)}\right| d s \\
& \leqslant|\beta|\left(\int_{T}^{t} \frac{y^{\prime 2}(s)}{y^{\gamma+1}(s)} d s\right)^{1 / 2}(t-T)^{1 / 2}
\end{aligned}
$$

By appealing to the simple inequality $(a+b)^{2} \leqslant 2 a^{2}+2 b^{2}$, we obtain from (24)

$$
\left|\frac{y^{1-\gamma}(t)}{t}\right| \leqslant 2 \beta^{2}\left(1-\frac{T}{t}\right)\left(\int_{T}^{t} \frac{y^{\prime 2}}{y^{\gamma+1}}\right)+2 \frac{y^{1-\gamma}(T)}{t} .
$$

Note that the integral in the above expression can be made arbitrarily small by choosing $T$ sufficiently large according to (22) and after fixing $T$, we also note that the last term tends to zero as $t \rightarrow \infty$. Hence (23) follows from (25). Let $c$ be the positive constant so that (21) is oscillatory. By (23), we can choose $t_{1} \geqslant t_{0}$ such that $y^{\gamma-1}(t) \geqslant c / \gamma t$ for all $t \geqslant t_{1}$. Then an application of the Picone-Sturm Theorem to (16) yields the desired conclusion as before. This completes the proof. 
Many well-known linear oscillation criteria are stated only for the equation $v^{\prime \prime}+a(t) v=0$, i.e. (8) with $p(t) \equiv 1$. In order to apply Theorem 2 we can transform (2) by the familiar Liouville transformation, $t=e^{s}$ and $z(s)=z(t)$, to

$$
z^{\prime \prime}(s)+e^{s} a\left(e^{s}\right) z(s)=0, \quad s \geqslant 0,
$$

where prime denotes differentiation with respect to $s$. Clearly, the oscillatory character of (21) and (26) remains the same. Theorem 2 can thus be restated as follows. Under appropriate conditions on $a(t)$, if (26) is weakly oscillatory, then equation (3) is 'strongly' oscillatory.

Some recent linear oscillation results, especially those in Kwong and Zettl [24, 25], apply directly to (21).

EXAmple 2. Consider $a(t)=t^{\lambda} \sin t$, when $\lambda=0$. This classic example for the linear equation, i.e. (3) with $\gamma=1$, was first resolved to be oscillatory by Yelchin [43] using explicit Fourier series expansion. The well-known extension of the Fite-Wintner Oscillation Theorem [37] by Hartman [14] also covers this case, so do results of Markus and Moore [27], Willett [35] and Komkov [19]. For the Emden-Fowler equation, this was settled by a recent paper of Butler [6]. We refer to Kwong and Zettl [25] where it was shown that the linear equation

$$
\left(t z^{\prime}(t)\right)^{\prime}+\phi(t) z(t)=0, \quad t \in[0, \infty),
$$

is strongly oscillatory where $\phi(t)$ is a periodic function of period $T$ which has zero mean value, i.e. $\int_{0}^{T} \phi(t) d t=0$. This result coupled with Theorem 2 gives an alternative solution to the question of oscillation of the Emden-Fowler equation with periodic coefficient. A closer look at Butler's Theorem reveals that it can be shown to follow from Theorem 2. The verification is rather complicated and we choose to omit such details. However, we note that as a simple consequence of Sturm's Comparison Theorem, every linear equation $\left(t z^{\prime}\right)^{\prime}+a(t) z=0$ is strongly oscillatory if $a(t) \geqslant \phi(t)$, a nonzero periodic function of mean value zero. Hence by Theorem 2 , equation (3) is oscillatory for such coefficients $a(t)$. This does not follow from Butler's result, one hypothesis of which requires $a(t)$ to be bounded.

REMARK 1. In another paper, Kwong and Zettl [24, Theorem 2] shows that (21) is weakly oscillatory if the function $\bar{A}(t)=\int_{0}^{t} a(s) d s$ satisfies the following condition:

There exists an increasing sequence of numbers $\left\{\lambda_{n}\right\}, \lambda_{n} \rightarrow \infty, n \rightarrow \infty$ such that

$$
\liminf _{n \rightarrow \infty} \lambda_{n} \int_{S_{n}} \frac{d t}{t}>0
$$

where $S_{n}$ is the set $\left\{t \in[0, \infty): \bar{A}(t) \geqslant \lambda_{n}\right\}$. Thus, under the same condition on $\bar{A}(t)$ and condition (20), equation (3) is oscillatory. In particular, if for every $\lambda>0$, $\int_{S_{\lambda}} d t / t=\infty$, where $S_{\lambda}=\{t \in[0, \infty): \bar{A}(t) \geqslant \lambda\}$, then (1) is oscillatory. If, however, for some $\lambda>0, \int_{S_{\lambda}} d t / t<\infty$ the above condition $(*)$ is still satisfied when there exists some $\alpha \in(0,1)$ such that

$$
\int_{S} \frac{\bar{A}^{\alpha}(t)}{t} d t=\infty
$$


see [24, Corollary 3]. Examples of $a(t)$ that satisfy the above criterion are

(i) $a(t)=1+\sin t+t \cos t$ with $\bar{A}(t)=t(1+\sin t)$.

(ii) Let $\left\{t_{n}\right\}$ be an increasing sequence with $t_{n+1}-t_{n}>2 \pi$ for all $n=1,2,3, \ldots$. Define

$$
a(t)= \begin{cases}t_{n} \sin \left(t-t_{n}\right), & t \in\left[t_{n}, t_{n}+\pi\right], \\ \text { arbitrary, } & \text { otherwise }\end{cases}
$$

and require that condition (20) be satisfied.

REMARK 2. In [25], it was shown that (21) is strongly oscillatory if there exist two numbers $\lambda_{1}<\lambda_{2}$ such that

$$
\int_{0}^{\infty} \frac{1}{t}\left[\bar{A}(t)-\lambda_{2}\right]_{+}^{2} d t=\int_{0}^{\infty} \frac{1}{t}\left[\bar{A}(t)-\lambda_{1}\right]_{-}^{2} d t=\infty,
$$

where $[g(t)]_{+}=\max \{g(t), 0\}$ and $[g(t)]_{-}=\max \{-g(t), 0\}$ for any function $g(t)$. Thus, under the same conditions on $\bar{A}(t)$ plus condition (20), equation (3) is oscillatory. In fact much more general results have been established in [25]. We refer the readers to the original paper for these, as well as examples of $a(t)$ satisfying these conditions.

REMARK 3. Theorem 2 also allows immediate translation of certain known linear oscillation criteria to the Emden-Fowler equation (3). We list just one of such applications as an example.

COROLLARY 4. Let ta $(t)$ be either bounded above or below and satisfy

$$
+\infty \geqslant \limsup _{T \rightarrow \infty} \int_{0}^{T} a(t) d t>\liminf _{T \rightarrow \infty} \int_{0}^{T} a(t) d t>-\infty .
$$

Then (3) is oscillatory.

This result follows from an application to (26) of a linear result by Olech, Opial and Wazewski (see [36, Corollary 5.2]). A weaker version of the Olech, Opial and Wazewski result was given earlier by Petropavlovskaya [29]. For a recent generalization of this linear oscillation criterion see Kwong and Zettl [24]. It is also a consequence of the results in [25].

REMARK 4. Recently Svatoslav [32] showed that if $\nu^{\prime \prime}+\lambda \phi(t) \nu=0$ where $\phi(t)$ is periodic with period $T$ and is oscillatory for all $\lambda \neq 0$, then $\phi(t)$ must have mean value zero, i.e. $\int_{0}^{T} a(t) d t=0$. For the nonlinear equation (3), the analogous problem has been studied by Butler, see [6].

4. In this section, we consider conditions on $a(t)$ applicable to so-called wildly oscillatory coefficients, an example being $a(t)=t^{\lambda} \sin t$ with $\lambda>0$. Here condition (20) fails to hold. The study of linear oscillation for such classes of $a(t)$ was the motivation for summability results using weighted averages by Coles and Willett [10], Willett [35,36]; see also Coppel [11] and recent results by Kwong and Zettl [25]. For the Emden-Fowler equation, the question of oscillation was only very recently settled by Butler [8] with a rather special method, based on Willett's idea of weighted averages. Unfortunately, as the author admits himself, his result does not allow ready extension to the more general equation (1). For the sublinear equation (3), 
$0<\gamma<1$, the result of Kamenev [16] would cover all cases of $t^{\lambda} \sin t, \lambda>0$. For more recent results applicable to sublinear equations see [21, 22]. We shall therefore confine our discussion in this section primarily to the superlinear equation when $\gamma>1$.

We shall show that condition (20) plus either condition $(*)$ or $(* *)$ mentioned in the last section remain sufficient for the oscillation of (3) with $\gamma>1$ if the function $\bar{A}(t)=\int_{0}^{t} a(s) d s$ is replaced by a more general function $\mathbb{Q}(t)=\int_{0}^{t} a(s) / \phi(s) d s$, where $\phi$ satisfies

$$
\phi^{\prime 2}(t) \geqslant \phi(t) \phi^{\prime \prime}(t) \text { for all large } t .
$$

Note that condition (28) is equivalent to the requirement that $\phi^{\prime} / \phi$ is nonincreasing for all large $t$. Examples of $\phi$ satisfying (28) are $t^{\alpha}, e^{\alpha t}, t^{\alpha} e^{\mu t},(\ln t)^{\alpha}, \exp \left(t^{\varepsilon}\right), \alpha>0$, $\mu>0$, and $0<\varepsilon<1$. Also, if $\phi$ satisfies (28), so does $\phi^{\alpha}$ for any $\alpha>0$. Thus, this proves that (3) is oscillatory when the coefficient $a(t)=t^{\lambda} \sin t, e^{\alpha t} \sin t$, and $e^{t} \sin e^{t}$, the so-called wildly oscillatory cases. Our main result is the following.

THEOREM 3. Suppose that there exists a $C^{2}$-nondecreasing positive function $\phi$ satisfying (28) and $a(t)$ satisfies

$$
Q(t)=\int_{0}^{t} \frac{a(s)}{\phi(s)} d s \geqslant-L>-\infty .
$$

If $\mathcal{Q}(t)$ satisfies either condition $(*)$ or $(* *)$, given in Remarks 1 and 2 of the last section, then (3) is oscillatory with $\gamma>1$.

We begin with a lemma establishing a stronger assertion than (23) that will be needed in the proof of Theorem 3.

LEMMA 1. Let $\phi$ be a $C^{2}$ positive and nondecreasing function satisfying

$$
\phi^{\prime 2}(t) \geqslant \frac{\gamma+1}{\gamma+2} \phi(t) \phi^{\prime \prime}(t)
$$

If in addition condition (29) is satisfied, then for every nonoscillatory solution $y(t)$ of (3) with $\gamma>1$, we have

$$
\lim _{t \rightarrow \infty}\left[t \phi(t) y^{\gamma-1}(t)\right]^{-1}=0
$$

Proof. Introduce the change of dependent variable $y(t)=\omega(t) / \phi^{\alpha}(t)$ where $\alpha=(\gamma-1)^{-1}>0$. Without loss of generality, we may assume $y(t)>0$ for all $t$. Equation (3) then transforms into the following:

$$
\omega^{\prime \prime} / \omega^{\gamma}-2 \alpha \phi^{\prime} \omega^{\prime} / \phi \omega^{\gamma}+g+a / \phi=0
$$

where

$$
g=\frac{\alpha\left[(\alpha+1) \phi^{\prime}-\phi \phi^{\prime \prime}\right]}{\phi^{2} \omega^{\gamma-1}}
$$


Integrating (32) from 0 to $t$, we obtain

$$
\begin{aligned}
-\frac{\omega^{\prime}(t)}{\omega^{\gamma}(t)} & =-\frac{\omega^{\prime}(0)}{\omega^{\gamma}(0)}-\int_{0}^{t} \frac{2 \alpha \phi^{\prime} \omega^{\prime}}{\phi \omega^{\gamma}}+\int_{0}^{t} \frac{a}{\phi}+\int_{0}^{t} g+\int_{0}^{t} \frac{\gamma \omega^{\prime 2}}{\omega^{\gamma+1}} \\
& =A_{1}(t)+\frac{2 \alpha^{2} \phi^{\prime}(t)}{\phi(t) \omega^{\gamma-1}(t)}+\int_{0}^{t} \frac{2 \alpha^{2}}{\omega^{\gamma-1}}\left(-\left(\frac{\phi^{\prime}}{\phi}\right)^{\prime}\right)+\int_{0}^{t} g+\int_{0}^{t} \frac{\gamma \omega^{\prime 2}}{\omega^{\gamma+1}}
\end{aligned}
$$

where

$$
A_{1}(t)=-\frac{\omega^{\prime}(0)}{\omega^{\gamma}(0)}+\int_{0}^{t} \frac{a}{\phi}-\frac{2 \alpha^{2} \phi^{\prime}(0)}{\phi(0) \omega^{\gamma-1}(0)} .
$$

By (30), we have

$$
\left[\frac{2 \alpha^{2}}{\omega^{\gamma-1}}\left(-\frac{\phi^{\prime}}{\phi}\right)^{\prime}+g\right] \geqslant 0,
$$

so we may drop the second, third and fourth terms on the right-hand side of (33) and obtain the following inequality:

$$
-\frac{\omega^{\prime}(t)}{\omega^{\gamma}(t)} \geqslant A_{1}(t)+\gamma \int_{0}^{t} \frac{\omega^{\prime 2}(s)}{\omega^{\gamma+1}(s)} d s .
$$

Condition (29) ensures that $A_{1}(t)$ is bounded below. If $\int_{0}^{\infty} \omega^{\prime 2} / \omega^{\gamma+1}=\infty$, then for sufficiently large $t,(34)$ becomes for some $t_{0} \geqslant 0$,

$$
-\frac{\omega^{\prime}(t)}{\omega^{\gamma}(t)} \geqslant m+\gamma \int_{0}^{t} \frac{\omega^{\prime 2}(s)}{\omega^{\gamma+1}(s)} d s,
$$

where $m>0$. Now we can apply our earlier result on integral inequality, see [23, Lemma 2], to (35) and obtain $\omega^{\prime}(t) \leqslant-m \omega^{\gamma}\left(t_{0}\right)<0$, contradicting the fact that $y(t)$ is nonoscillatory. On the other hand, if $\int_{0}^{\infty} \omega^{\prime 2} / \omega^{\gamma+1}<\infty$, then we can repeat the same argument in Theorem 2 to conclude that $\lim _{t \rightarrow \infty}\left(t \omega^{\gamma-1}(t)\right)^{-1}=0$, which is the desired result (31).

Proof of Theorem 3. Notice that (28) implies (30); thus the lemma applies. Let $y$ be a nonoscillatory solution of (3) and proceed in the same manner as in the proof of the lemma above. Since $\phi^{\prime} \geqslant 0$ and $\left(\phi^{\prime} / \phi\right)^{\prime} \leqslant 0$, we may deduce from (33) the following inequality:

$$
-\frac{\omega^{\prime}(t)}{\omega^{\gamma}(t)} \geqslant A_{1}(t)+\int_{0}^{t} g+\gamma \int_{0}^{t} \frac{\omega^{\prime 2}}{\omega^{\gamma+1}} .
$$

By (29), $A_{1}(t)$ is bounded below and so $\int_{0}^{t} g$ and $\int_{0}^{t} \omega^{\prime 2} / \omega^{\gamma+1}$ must both converge. Otherwise, we can repeat the argument on (36) commencing from (35) and conclude that $\omega^{\prime}(t) \leqslant-m \omega^{\gamma}\left(t_{0}\right)<0$ for some appropriate positive constants $m$ and $t_{0} \geqslant 0$, contradicting the fact that $y$ is nonoscillatory. From $\int_{0}^{\infty} g<\infty$, we obtain

$$
\int_{0}^{\infty} g=\int_{0}^{\infty} \frac{\alpha\left[\phi^{\prime 2}-\phi \phi^{\prime \prime}\right]+\alpha^{2} \phi^{\prime 2}}{\phi^{2} \omega^{\gamma-1}} \geqslant \int_{0}^{\infty} \frac{\alpha^{2} \phi^{\prime 2}}{\phi^{2} \omega^{\gamma-1}} .
$$


An application of Schwarz inequality yields

$$
\int_{0}^{\infty}\left|\frac{\phi^{\prime} \omega^{\prime}}{\phi \omega^{\gamma}}\right| \leqslant\left(\int_{0}^{\infty} \frac{\phi^{\prime 2}}{\phi^{2} \omega^{\gamma-1}}\right)^{1 / 2}\left(\int_{0}^{\infty} \frac{\omega^{\prime 2}}{\omega^{\gamma+1}}\right)^{1 / 2}<\infty .
$$

We claim that (37) implies (a condition similar to (23) for $\omega(t)$ instead of $y(t)$ )

$$
\lim _{t \rightarrow \infty} \frac{\phi^{\prime}(t)}{\phi(t) \omega^{\gamma-1}(t)}=0 \text {. }
$$

First, suppose that $\int_{0}^{\infty} \phi^{\prime}(s) / \phi(s) d s<\infty$, which upon integration by parts yields

$$
\frac{t \phi^{\prime}(t)}{\phi(t)}-\int_{0}^{t} s\left(\frac{\phi^{\prime}(s)}{\phi(s)}\right)^{\prime} d s=\int_{0}^{t} \frac{\phi^{\prime}(s)}{\phi(s)} d s \leqslant \int_{0}^{\infty} \frac{\phi^{\prime}}{\phi}<\infty
$$

and by (28), we obtain that $t \phi^{\prime} / \phi$ is bounded above; hence (39) follows from (31). Next, we assume that $\int_{0}^{\infty} \phi^{\prime}(s) / \phi(s) d s=\infty$ (hence $\lim _{t \rightarrow \infty} \phi(t)=\infty$, which is the more interesting case).

Integrating by parts, we obtain the following equation:

$$
\frac{\phi^{\prime}(t)}{\phi(t) \omega^{\gamma-1}(t)}-\frac{\phi^{\prime}\left(t_{0}\right)}{\phi\left(t_{0}\right) \omega^{\gamma-1}\left(t_{0}\right)}=\int_{t_{0}}^{t}\left(\frac{\phi^{\prime}}{\phi}\right)^{\prime} \frac{1}{\omega^{\gamma-1}}-(\gamma-1) \int_{t_{0}}^{t} \frac{\phi^{\prime} \omega^{\prime}}{\phi \omega^{\gamma}} .
$$

The last integral in the above equation converges on account of (38); and since the first integral is nonpositive, the integral either converges or diverges to infinity. In case it diverges, (40) shows that

$$
\lim _{t \rightarrow \infty} \frac{\phi^{\prime}(t)}{\phi(t) \omega^{\gamma-1}(t)}=-\infty
$$

which contradicts $\phi^{\prime} \geqslant 0, \phi, \omega>0$. Otherwise, (40) yields that $\lim _{t \rightarrow \infty} \phi^{\prime}(t) /\left(\phi(t) \omega^{\gamma-1}(t)\right)$ exists and if the limit is not zero it must be positive. Suppose for large $t$, we have $\phi^{\prime}(t) / \phi(t) \omega^{\gamma-1}(t)>\beta>0$, for some $\beta$, which upon being substituted in (37) yields $\int^{\infty} \phi^{\prime} / \phi<\infty$, a desired contradiction.

The importance of (39) is that the function

$$
A^{*}(t)=\mathbb{Q}(t)-\frac{2 \alpha^{2} \phi^{\prime}(0)}{\phi(0) \omega^{\gamma-1}(0)}+\frac{2 \alpha^{2} \phi^{\prime}(t)}{\phi(t) \omega^{\gamma-1}(t)}
$$

again satisfies conditions $(*)$ or $(* *)$, which hold by the hypothesis on $Q(t)$. Define

$$
h(t)=\frac{2 \alpha^{2}}{\omega^{\gamma-1}(t)}\left(-\left(\frac{\phi^{\prime}(t)}{\phi(t)}\right)^{\prime}\right)+g(t) \geqslant 0,
$$

with which we can simplify (33) to

$$
\frac{-\omega^{\prime}(t)}{\omega^{\gamma}(t)}=\frac{-\omega^{\prime}(0)}{\omega^{\gamma}(0)}+A^{*}(t)+\int_{0}^{t} h(s) d s+\gamma \int_{0}^{t} \frac{\omega^{\prime 2}(s)}{\omega^{\gamma+1}(s)} d s .
$$

Once again, we return to the proof of Theorem 2 and deduce that the existence of a solution to the integral equation (42) implies the nonoscillation of the linear equation

$$
\left(t z^{\prime}(t)\right)^{\prime}+\left(a^{*}(t)+h(t)\right) z(t)=0
$$


where $a^{*}(t)=\left[A^{*}(t)\right]^{\prime}$. Since $h \geqslant 0$, by the Sturm Comparison Theorem, we know that the linear equation $\left(t \nu^{\prime}\right)^{\prime}+a^{*} \nu=0$ is nonoscillatory. This contradicts the assertion that $A^{*}(t)$ satisfies condition $(*)$ or $(* *)$ for oscillation. The proof is now complete.

REMARK 5. Suppose that (3) satisfies the hypotheses of Theorem 3 and

$$
y^{\prime \prime}(t)+a_{1}(t)|y(t)|^{\gamma} \operatorname{sgn} y(t)=0, \quad \gamma>1,
$$

is another superlinear equation with the same constant $\gamma$, and if $a_{1}(t) \geqslant a(t)$ for all large $t$. Then (44) will correspond to a linear equation of the form (43) with $a^{*}$ replaced by some function $a_{1}^{*}(t) \geqslant a^{*}(t)$ for all large $t$. The Picone-Sturm Comparison Theorem then implies the oscillation of this linear equation. Thus (44) is also oscillatory. This widens the applicability of Theorem 3 because although $a(t)$ may satisfy $(* *), a_{1}(t)$ need not do so.

REMARK 6. The following is an alternative formulation of the improved version (see Remark 5) of Theorem 3.

Theorem 3'. Suppose that $\phi \in C^{2}[0, \infty)$ satisfies (28) and there is a function $\tilde{a}(t) \leqslant \bar{a}(t)$, and $\tilde{A}(t)=\int_{0}^{t} \tilde{a}(s) d s$ satisfies (29) and either condition $(*)$ or $(* *)$. Then the equation

$$
y^{\prime \prime}(t)+\phi(t) \bar{a}(t)|y(t)|^{\gamma} \operatorname{sgn} y=0, \quad \gamma>1,
$$

is oscillatory on $[0, \infty)$.

EXAMPLE 3. Consider $\bar{a}(t) \geqslant \tilde{a}(t)=\psi(t)$, when $\psi(t)$ is a periodic function of period $T$ satisfying $\int_{0}^{T} \psi(t) d t=0$. Then the equation

$$
y^{\prime \prime}(t)+\phi^{\lambda}(t) \bar{a}(t) \mid y(t) \gamma^{\gamma} \operatorname{sgn} y=0, \quad \gamma>1,
$$

is oscillatory for all $\lambda>0$, and $\phi(t)$ is any one of the following functions: $t, e^{t}, t e^{\mu t}$ $(\mu>0),(\ln t)^{\mu}, \exp \left(t^{\varepsilon}\right)(0<\varepsilon<1)$.

EXAMPLE 4. Consider $a(t)=e^{\lambda t} \cos e^{t}, \lambda>1$ in (3) with $\gamma>1$. Here we let $\phi(t)=e^{(\lambda-1) t}$ and $\tilde{a}(t)=\bar{a}(t)=e^{t} \cos e^{t}$. Thus, $\tilde{A}(t)=\sin e^{t}$ which is clearly bounded below and also satisfies condition $(* *)$. Hence, (3) is oscillatory, by Theorem 3'.

REMARK 7. In view of Lemma 1 it is obvious that the following result of the form of Theorem 2 holds:

Suppose there exists a $C^{2}$ positive and nondecreasing function satisfying (30) such that (29) also holds. If the linear equation

$$
\left(t \phi(t) z^{\prime}(t)\right)^{\prime}+a(t) z(t)=0, \quad t \in[0, \infty),
$$

is weakly oscillatory, then (3) with $\gamma>1$ is strongly oscillatory.

The proof of Theorem 3 contains a kind of perturbation result for linear oscillation. Consider the linear equation $\left(t z^{\prime}\right)^{\prime}+q(t) z=0$ or more generally

$$
\left(p(t) z^{\prime}\right)^{\prime}+q(t) z=0
$$

which is oscillatory (strongly oscillatory). The essential part of the proof is that the following perturbed equation

$$
\left(p(t) z^{\prime}\right)^{\prime}+(q(t)+\eta(t)) z=0
$$


is also oscillatory (strongly oscillatory), where $\eta(t)$ is continuous such that $\lim _{t \rightarrow \infty} \int_{0}^{t} \eta(s) d s$ exists and is finite. This is the case when $p(t)=t$ and the function $Q(t)=\int_{0}^{t} q(s) d s$ satisfies condition $(*)$ or $(* *)$.

Let us call (46) (strongly) stably oscillatory of the first kind or simply (strongly) S1-oscillatory when the above is true.

We also define a second and stronger kind of stable oscillation by requiring that (47) remain oscillatory (strongly oscillatory) if $\int_{0}^{t} \eta(s) d s$ is merely assumed to be bounded. In this case let us call (46) (strongly) S2-oscillatory. When $p(t)=t$ and $q(t)$ satisfies (*) then (46) is strongly S2-oscillatory. This is also true if $p(t)=t$ and $(* *)$ is satisfied for some $\lambda_{1}$ but for all $\lambda_{2}>\lambda_{1}$, for instance when $q(t)=h(t) \sin t$, where $h(t)$ is a continuous nondecreasing function such that $\lim _{t \rightarrow \infty} h(t)=\infty$.

Theorem 3 can now be reformulated in the more abstract form.

Theorem $3^{\prime \prime}$. Suppose that $\phi \in C^{2}[0, \infty)$ satisfies (28) and $A(t)=\int_{0}^{t} \bar{a}(s) d s$ satisfies (29) and the linear equation $\left(t z^{\prime}\right)^{\prime}+\bar{a}(t) z=0$ is strongly S1-oscillatory, then

$$
y^{\prime \prime}(t)+\phi(t) \bar{a}(t)|y(t)|^{\gamma} \operatorname{sgn} y=0, \quad \gamma>1, \quad t \in[0, \infty),
$$

is oscillatory on $[0, \infty)$.

The usefulness of the concepts of stable oscillations defined above will be further expounded in the next section when sublinear equations are studied. It is thus a pertinent problem to seek sufficient conditions for the two kinds of stable oscillations of (46) not contained in those of $(*)$ and $(* *)$.

5. In this section we consider only sublinear equations, (3) with $0<\gamma<1$. As in $[21,22]$ we make use of weight functions $\phi$ in $C^{2}[0, \infty)$ such that

$$
\phi^{\prime}(t) \geqslant 0, \quad \phi^{\prime \prime}(t) \leqslant 0, \quad t \geqslant 0 .
$$

In [21], it is shown that if

$$
\limsup _{T \rightarrow \infty} \frac{1}{T} \int_{0}^{T} \int_{0}^{t} \phi^{\gamma}(s) a(s) d s d t=\infty
$$

then (3) with $0<\gamma<1$ is oscillatory. In [22] a sufficient criterion is given in case

$$
\lim _{T \rightarrow \infty} \frac{1}{T} \int_{0}^{T} \int_{0}^{t} \phi^{\gamma}(s) a(s) d s d t \quad \text { exists and is finite. }
$$

Upon applying the two results to the particular example

$$
y^{\prime \prime}(t)+t^{\lambda} \sin t|y(t)|^{\gamma} \operatorname{sgn} y=0
$$

$0<\gamma<1, t \in[0, \infty)$ and $\lambda \in(-\infty, \infty)$, we were able to show oscillation when $\lambda>-\gamma$. A further result in [22] gives nonoscillation when $\lambda<-\gamma$. The case $\lambda=-\gamma$ was then unsettled. We shall prove below that we have oscillation in this limiting case.

Here we give a linearization theorem for those coefficients $a(t)$ satisfying

$$
\liminf _{T \rightarrow \infty} \frac{1}{T} \int_{0}^{T} \int_{0}^{t} \phi^{\gamma}(s) a(s) d s d t>-\infty
$$

This covers a much wider class of coefficient than that considered in [22]. 
LEMma 2. Suppose that there exists a $C^{2}$ function $\phi$ satisfying (48) and (52), and (3) has a solution $y>0$ on some half ray $[b, \infty), b>0$. Let

$$
u(t)=[y(t) / \phi(t)]^{\gamma}, \quad t>b,
$$

and

$$
\beta=1 / \gamma>1
$$

Then

$$
\limsup _{t \rightarrow \infty} \frac{\phi(t) u^{\beta-1}(t)}{t}<\infty
$$

and

$$
\int_{b}^{\infty} \phi(s) u^{\beta-3}(s) u^{\prime 2}(s) d s<\infty .
$$

If furthermore (50) holds, then

$$
\lim _{t \rightarrow \infty} \frac{\phi(t) u^{\beta-1}(t)}{t} \text { exists and is finite. }
$$

Proof. The following identity can be easily verified

$$
-\left(\phi u^{\beta-1}\right)^{\prime \prime}=\frac{\beta-1}{\beta} a \phi^{\gamma}+(\beta-1) \phi u^{\beta-3} u^{\prime 2}+\frac{1}{\beta} \phi^{\prime \prime} u^{\beta-1} .
$$

Integrate this twice and then divide by $T$ to obtain

$$
\begin{aligned}
& -\frac{\phi(T) u^{\beta-1}(T)}{T}=A+\frac{B}{T}+\frac{\beta-1}{\beta T} \int_{0}^{T} \int_{0}^{t} \phi^{\gamma}(s) a(s) d s d t \\
& \quad+\frac{\beta-1}{T} \int_{0}^{T} \int_{0}^{t} \phi(s) u^{\beta-3}(s) u^{\prime 2}(s) d s d t+\frac{1}{\beta T} \int_{0}^{T} \int_{0}^{t}\left[-\phi^{\prime \prime}(s)\right] u^{\beta-1}(s) d s d t
\end{aligned}
$$

where $A$ and $B$ are integration constants. Suppose (56) does not hold. It follows that the second integral on the right-hand side of (59) tends to infinity as $T \rightarrow \infty$. Since the last term in (59) is nonnegative and (52) holds, the right-hand side of (59) tends to infinity as $T \rightarrow \infty$. This is impossible due to the form of the left-hand side. Thus (56) must hold. It follows that

$$
\begin{aligned}
\lim _{T \rightarrow \infty} \frac{\beta-1}{T} \int_{0}^{T} \int_{0}^{t} \phi(s) u^{\beta-3}(s) u^{\prime 2}(s) d s d t \\
\quad=(\beta-1) \int_{0}^{\infty} \phi(s) u^{\beta-3}(s) u^{\prime 2}(s) d s<\infty
\end{aligned}
$$

Similar arguments when applied to the last integral of (59) lead to

$$
\lim _{T \rightarrow \infty} \frac{1}{\beta T} \int_{0}^{T} \int_{0}^{t}\left[-\phi^{\prime \prime}(s)\right] u^{\beta-1}(s) u^{\beta-1}(s) d s d t=\frac{1}{\beta} \int_{0}^{\infty}\left[-\phi^{\prime \prime}(s)\right] u^{\beta-1}(s) d s<\infty .
$$

Taking the limits of the two sides of (59) now yields (55) as well as (57) under the additional assumption (50). 
REMARK 8. From the proof, it is easy to see that in order to deduce (56) we need only assume instead of (52) the weaker condition

$$
\limsup _{T \rightarrow \infty} \frac{1}{T} \int_{0}^{T} \int_{0}^{t} \phi^{\gamma}(s) a(s) d s d t>-\infty,
$$

which however is not sufficient to yield (55).

THEOREM 4. Suppose there exists a function $\phi \in C^{2}[0, \infty)$ satisfying (48) such that the coefficient $a(t)$ satisfies (52). If the linear equation

$$
\left(t z^{\prime}(t)\right)^{\prime}+\phi^{\gamma}(t) a(t) z(t)=0, \quad t \in[0, \infty),
$$

is strongly S2-oscillatory, then (3) with $0<\gamma<1$ is oscillatory.

Proof. After integrating (58) once, we carry out the differentiation on the left-hand side to obtain

$$
\begin{aligned}
& -(\beta-1) \phi(t) u^{\beta-2}(t) u^{\prime}(t)=A+\phi^{\prime}(t) u^{\beta-1}(t) \\
& +\frac{\beta-1}{\beta} \int_{0}^{t} \phi^{\gamma}(s) a(s) d s+(\beta-1) \int_{0}^{t} \phi(s) u^{\beta-3}(s) u^{\prime 2}(s) d s \\
& +\frac{1}{\beta} \int_{0}^{t}\left[-\phi^{\prime \prime}(s)\right] u^{\beta-1}(s) d s .
\end{aligned}
$$

Since $\phi$ is a positive concave function, $t \phi^{\prime}(t) / \phi(t) \leqslant 1$. Hence by (55), $\phi^{\prime}(t) u^{\beta-1}(t) \leqslant \phi(t) u^{\beta-1}(t) / t \leqslant M$ for some positive constant $M$ and for all $t \geqslant b$. Let $\eta(t)=\left(\phi^{\prime}(t) u^{\beta-1}(t)\right)^{\prime}$. Then

$$
\limsup _{t \rightarrow \infty}\left|\int_{b}^{t} \eta(s) d s\right|<\infty
$$

Equation (61) becomes

$$
\begin{aligned}
-r(t)= & B+\int_{b}^{t}\left(\left(\frac{\beta-1}{\beta}\right) \phi^{\gamma} a+\eta+\frac{1}{\beta}\left[-\phi^{\prime \prime}\right] u^{\beta-1}\right) \\
& +\int_{b}^{t} \frac{r^{2}(s)}{(\beta-1) \phi(s) u^{\beta-1}(s)} d s
\end{aligned}
$$

with $r=(\beta-1) \phi u^{\beta-2} u^{\prime}$. Thus the linear equation $\left(\phi(t) u^{\beta-1}(t) Z^{\prime}(t)\right)^{\prime}$

$$
+\frac{1}{\beta-1}\left(\left(\frac{\beta-1}{\beta}\right) \phi^{\gamma}(t) a(t)+\eta(t)+\frac{1}{\beta}\left[-\phi^{\prime \prime}(t) u^{\beta-1}(t)\right]\right) Z(t)=0
$$

is nonoscillatory. Since $\phi(t) u^{\beta-1}(t) \leqslant M t$ and $-\left[\phi_{u}^{\prime \prime \beta-1}\right]$ is positive, the Picone-Sturm Comparison Theorem yields the nonoscillation of the linear equation

$$
M\left(t z^{\prime}(t)\right)^{\prime}+\frac{1}{\beta-1}\left(\left(\frac{\beta-1}{\beta}\right) \phi^{\gamma}(t) a(t)+\eta(t)\right) z(t)=0 .
$$

This contradicts the hypothesis that $(60)$ is strongly S2-oscillatory. 
THEOREM 5. Suppose there exists a function $\phi \in C^{2}[0, \infty)$ satisfying (48) and

$$
\lim _{t \rightarrow \infty} \frac{t \phi^{\prime}(t)}{\phi(t)} \quad \text { exists and is finite }
$$

such that the coefficient a(t) satisfies (50). If (50) is strongly S1-oscillatory, then (3) with $0<\gamma<1$ is oscillatory.

Suppose instead of (63), $\phi$ satisfies the stronger condition

$$
\lim _{t \rightarrow \infty} \frac{t \phi^{\prime}(t)}{\phi(t)}=0
$$

and the coefficient $s(t)$ satisfies (52). If (60) is strongly S1-oscillatory, then (3) with $0<\gamma<1$ is oscillatory.

Proof. We proceed as in the proof of Theorem 4. Notice that (63) and (57), or (64) and (55) imply that

$$
\lim _{t \rightarrow \infty} \phi^{\prime}(t) u^{\beta-1}(t)=\left(\lim _{t \rightarrow \infty} \frac{t \phi^{\prime}(t)}{\phi(t)}\right)\left(\lim _{t \rightarrow \infty} \frac{\phi(t) u^{\beta-1}(t)}{t}\right) \text { exists. }
$$

Thus $\lim _{t \rightarrow \infty} \int_{b}^{t} \eta(s) d s$ exists and is finite. The rest of the proof remains unchanged.

Examples of $\phi$ for which (48) and (63) are satisfied are $\phi(t)=t^{\mu}, 0<\mu<1 ; \ln ^{\mu} t$, $0<\mu<1$.

EXAmPle 5. The coefficient $a(t)=t^{\lambda} \sin t$ satisfies (50) when $-\gamma \leqslant \lambda<1-\gamma$ and $\phi(t)=t$. As already noted, the linear equation (60) is strongly S1-oscillatory. Hence by Theorem $5,(3)$ is oscillatory. The classification of (51) is thus finally completed. As an illustration of how Theorem 4 applies, consider the case $\lambda=1-\gamma$ and $\phi(t)=t$. Then (52) holds instead of (50). Equation (60) in this case is strongly S2-oscillatory and so (51) is oscillatory. When $\lambda>1-\gamma$, then (49) holds with $\phi(t) \equiv t$, so (51) is oscillatory by our earlier result [21].

The particular choice of weight function $\phi \equiv 1$ deserves special mention. By the remark after Lemma 2 we see that

$$
\limsup _{T \rightarrow \infty} \frac{1}{T} \int_{0}^{T} \int_{0}^{t} a(s) d s d t>-\infty
$$

is sufficient to yield

$$
\int_{0}^{\infty} u^{\beta-3}(s) u^{\prime 2}(s) d s<\infty .
$$

Returning to the variable $y$ instead of $u$, we see that (66) is equivalent to (22). As in the proof of Theorem 2, (22) implies (23), and the rest of the proof of Theorem 2 carries over. We thus have the following stronger version of Theorem 2 for the sublinear equation.

THEOREM 6. Suppose that (65) holds. If the linear equation $\left(t z^{\prime}(t)\right)^{\prime}+a(t) z(t)=0$ is weakly oscillatory, then (3) is oscillatory for $0<\gamma<1$.

That no stable oscillation is required of the linear comparison equation as a contrast to Theorems 4 and 5 can be explained by the fact that with the choice $\phi \equiv 1$, the term $\phi^{\prime} u^{\beta-1}$ in (61) is missing. 
Theorem 6 includes a result of Butler in [8], which states that

$$
-\infty \leqslant \liminf _{T \rightarrow \infty} \frac{1}{T} \int_{0}^{T} \int_{0}^{t} a(s) d s d t<\limsup _{T \rightarrow \infty} \frac{1}{T} \int_{0}^{T} \int_{0}^{t} a(s) d s d t \leqslant \infty
$$

implies that (3) is oscillatory with $0<\gamma<1$.

In fact (67) implies condition $(* *)$ which implies oscillation of the linear comparison equation as pointed out in [25] and so Theorem 6 applies.

\section{REFERENCES}

1. F. V. Atkinson, On second order nonlinear oscillation, Pacific J. Math. 5 (1955), 643-647.

2. J. H. Barrett, Oscillation theory of ordinary linear differential equations, Adv. in Math. 3 (1969), 415-509.

3. S. Belohorec, Oscillatory solutions of certain nonlinear differential equations of second order, Mat. Fyz. Časopis Sloven Akad. Vied. 11 (1961), 250-255. (Czech)

4. Two remarks on the properties of solutions of a nonlinear differential equation, Acta Fac. Rerum Natur. Univ. Comenian. Math. Publ. 22 (1969), 19-26.

5. G. J. Butler, On the oscillatory hehavior of a second order nonlinear differential equation, Ann. Mat. Pura Appl. 105 (1975), 73-92.

6. Oscillation theorems for a nonlinear analogue of Hill's equation, Quart. j. Math. Oxford Ser. (2) 27 (1976), 159-171.

7. Hille Wintner type comparison theorems for second order ordinary differential equations, Proc. Amer. Math. Soc. 76 (1979), 159-171.

8. Integral averages and the oscillation of second order ordinary differential equations, SIAM $\mathbf{J}$. Math. Anal. 11 (1980), 190-200.

9. W. J. Coles, Oscillation criteria for nonlinear second order equations, Ann. Mat. Pura Appl. 82 (1969), $123-134$.

10. W. J. Coles and D. Willett. Summability criteria for oscillation of second order linear differential equations, Ann. Mat. Pura Appl. 79 (1968), 391-398.

11. W. A. Coppel, Disconjugacy, Lecture Notes in Math., vol. 220, Springer-Verlag, Berlin, Heidelberg and New York, 1971.

12. L. Erbe, Oscillation theorems for second order nonlinear differential equations, Proc. Amer. Math. Soc. 24 (1970), $811-814$.

13. Oscillation criteria for second order nonlinear differential equations, Ann. Mat. Pura Appl. 94 (1972), 257-268.

14. P. Hartman, On non-oscillatory linear differential equations of second order, Amer. J. Math. 74 (1952), 389-400).

15. Ordinary differential equations, Wiley, New York, 1964.

16. I. V. Kamenev, Some specially nonlinear oscillation theorems, Mat. Zametki 10 (1971), 129-134.

17. I. T. Kamenev, Oscillation criteria related to averaging of solutions of ordinary differential equations of second order. Differencial'nye Uravnenija 10 (1974), 246-252. (Russian)

18. I. T. Kiguradze, $A$ note on the oscillation of solution of the equation $u^{\prime \prime}+a(t) u^{n} \operatorname{sgn} u=0$, Casopis Pěst. Mat. 92 (1967), 343-350.

19. V. Komkov, A technique for the detection of oscillation of second order ordinary differential equations, Pacific J. Math. 42 (1972), 105-115.

20. M. K. Kwong, On certain Riccati integral equations and second order linear oscillation, J. Math. Anal. Appl. 85 (1982), 315-330.

21. Man Kam Kwong and J. S. W. Wong, On an oscillation theorem of Belohorec, SIAM J. Math. Anal. 14 (1983),

22. . On the oscillation and nonoscillation of second order sublinear equations, Proc. Amer. Math. Soc. 85 (1982), 547-551.

23. An application of integral inequality to second order nonlinear oscillation, J. Differential Equations 46 (1982), 63-77.

24. Man Kam Kwong and A. Zettl, Differential and integral inequalities and second order linear oscillation, J. Differential Equations 45 (1982), 16-33.

25. , Asymptotically constant functions and second order linear oscillation, J. Math. Anal. Appl. (to appear). 
26. J. W. Macki and J. S. W. Wong, Oscillation criteria for linear second order ordinary differential equations, Proc. Amer. Math. Soc. 20 (1969), 67-72.

27. L. Markus and R. A. Moore, Oscillation and disconjugacy for linear differential equations with almost periodic coefficients, Acta Math. 96 (1956), 99-123.

28. H. Onose, Oscillation criteria for second order nonlinear differential equations, Proc. Amer. Math. Soc. 51 (1975), 67-73.

29. R. V. Petropavlovskaya, On oscillation of solutions of the equation $u^{\prime \prime}+p(x) u=0$, Dokl. Akad. Nauk SSSR (NS) 105 (1955), 29-31. (Russian)

30. C. R. Putnam, Note on some oscillation criteria, Proc. Amer. Math. Soc. 6 (1955), 950-952.

31. M. Rab, Kriterien fur die Oszillation der Lozungen der Differenzialgleichung $\left(p(x) y^{\prime}\right)^{\prime}+g(x) y=0$, Časopis Pěst. Mat. 84 (1959), 335-370; errata, ibid. 85 (1960), 91.

32. Stanek Svatoslav, $A$ note on the oscillation of solutions of the differential equation $v^{\prime \prime}=\lambda q(t) v$ with a periodic coefficient, Czechoslovak Math. J. 29 (1979), 318-323.

33. W. R. Utz, Properties of solutions of $u^{\prime \prime}+g(t) u^{2 n-1}=0$, Monatsh. Math. 66 (1962), 55-60.

34. P. Waltman, An oscillation criterion for a nonlinear second order equation, J. Math. Anal. Appl. 10 (1965), 439-441.

35. D. Willett, On the oscillatory behaviour of the solution of second order linear differential equations, Ann. Polon. Math. 21 (1969), 175-194.

36. , Classification of second order linear differential equations with respect to oscillation, Adv. in Math. 3 (1969), 594-623.

37. A. Wintner, A criterion of oscillatory stability, Quart. Appl. Math. 7 (1949), 115-117.

38. J. S. W. Wong, On second order nonlinear oscillation, Funkcial. Ekvac. 11 (1969), 207-234.

39. Oscillation and nonoscillation of solutions of second order linear differential equations with integrable coefficients, Trans. Amer. Math. Soc. 144 (1969), 197-215.

40. A second order nonlinear oscillation theorem, Proc. Amer. Math. Soc. 40 (1973), 487-491.

41. Oscillation theorems for second order nonlinear differential equations, Bull. Inst. Math. Acad. Sinica 3 (1975), 283-309.

42. __ On the generalized Emden-Fowler equation, SIAM Rev. 17 (1975), 339-360.

43. M. Yelchin, Sur le condition pour q'une solution d'un système linéaire du second ordre possede deux zeros, Dokl. Akad. Nauk SSSR 51 (1946), 573-576.

44. M. Zlamal, Oscillation criteria, Časopis Pěst. Mat. 75 (1950), 213-217.

Argonne National Laboratory, Argonne, Illinois 60439

DePartment of Mathematical SCIEnCES, Northern Illinois University, DeKalb, Illinois 60115

China Dyeing Works, Ltd., 819 Swire House, Hong Kong

DePartment of Mathematics, University of Hong Kong, Hong Kong 